\title{
JORDAN-VON NEUMANN TYPE CONSTANT AND FIXED POINTS FOR MULTIVALUED NONEXPANSIVE MAPPINGS
}

\author{
Zhanfei Zuo, Chunlei Tang, Xiaochun Chen and Liangwei Wang
}

Abstract. We give some sufficient conditions for the Domínguez-Lorenzo condition in terms of the Jordan-von Neumann type constant, and the coefficient of weak orthogonality. As a consequence, we obtain some sufficient conditions for normal structure and fixed point theorems for multivalued nonexpansive mappings. These fixed point theorems improve some previous results in the recently papers.

Mathematics subject classification (2010): Primary 47H10; Secondary 46B20.

Keywords and phrases: Multivalued nonexpansive mapping, fixed point, normal structure, Jordan-von Neumann type constant, coefficient of weak orthogonality.

\section{REFERENCES}

[1] J. Alonso And P. Martín, A counterexample to a conjecture of G. Zbăganu about the NeumannJordan constant, Rev. Roumaine Math. Pures Appl. 51 (2006), no. 2, 135-141.

[2] J. A. Clarkson, The von Neumann-Jordan constant for the Lebesgue spaces, Ann. of Math. (2) 38 (1937), no. 1, 114-115.

[3] S. Dhompongsa, P. Piraisanguun, And S. Saejung, Generalised Jordan-von Neumann constants and uniform normal structure, Bull. Austral. Math. Soc. 67 (2003) 225-240.

[4] S. Dhompongsa, A. Kaewkhao, and S. Tasena, On a generalized James constant, J. Math. Anal. Appl. 285 (2003) 419-435.

[5] S. Dhompongsa, A. Kaewcharoen, A. Kaewkhao, The Domínguez-Lorenzo condition and multivalued nonexpansive mappings, Nonlinear Anal. 64 (2006) 958-970.

[6] S. Dhompongsa, T. Domínguez Benavides, A. Kaewcharoen, A. Kaewkhao, B. PaNYANAK, The Jordan-von Neumann constant and fixed points for multivalued nonexpansive mappings, J. Math. Anal. Appl. 320 (2006) 916-927.

[7] J. GAO AND K.-S. LAU, On two classes of Banach spaces with uniform normal structure, Studia Math. 99 (1) (1991) 41-56.

[8] A. Jiménez-Melado, E. Llorens-Fuster, and S. Saejung, The von Neumann-Jordan constant, weak orthogonality and normal structure in Banach spaces, Proc. Amer. Math. Soc. 134 (2006) 355-364.

[9] A. Kaewkнаo, The James constant, the Jordan-von Neumann constant, weak orthogonality and fixed points for multivalued mappings, J. Math. Anal. Appl. 333 (2) (2007) 950-958.

[10] W. A. KIRK, A fixed point theorem for mappings which do not increase distances, Amer. Math. Monthly 72 (1965) 1004-1006.

[11] S. B. NADler JR., Multivalued contraction mappings, Pacific J. Math. 30 (1969) 475-488.

[12] S. SAEJung, On James and von Neumann-Jordan constants and sufficient conditions for the fixed point property, J. Math. Anal. Appl. 323 (2006) 1018-1024.

[13] B. Sims, Orthogonality and fixed points of nonexpansive maps, in: Proc. Centre Math. Anal. Austral. Nat. Univ, Austral. Nat. Univ, Canberra, (1988), 178-186.

[14] Y. TAKAhashi, Some geometric constants of Banach spaces-a unified approach, in: Banach and Function Spaces II, Proc. International Symposium (ISBFS 2006) held in Kitakyushu (Sept. 14-17, 2006), Edited by Mikio Kato and Lech Maligranda, Yokohama Publ., Yokohama 2008, 191-220. 
[15] C. S. YANG AND Y. M. WANG, Some properties on Jordan-von Neumann type constant of Banach spaces, Acta Mathematica Scientia, 2012, 32A (1): 212-221.

[16] G. ZBĂGANU, An inequality of $M$. Radulescu and $S$. Radulescu which characterizes the inner product spaces, Rev. Roumaine Math. Pures Appl. 47 (2) (2002), 253-257.

[17] Zhanfei Zuo, YunAn CUI, A coefficient related to some geometrical properties of Banach space, Journal of Inequalities and Applications, Volume 2009 (2009), Article ID 934321.

[18] Zhanfei Zuo, An Application of Zbăganu Constant in Fixed Point Theory, Acta Mathematica Vietnamica Volume 37, Number 1, 2012, 71-77.

[19] Zhanfei Zuo, The Ptolemy constant of absolute normalized norms on $R^{2}$, Journal of Inequalities and Applications, 2012, 1-14.

[20] Zhanfei Zuo, YunAN CUI, Some modulus and normal structure in Banach space, Journal of Inequalities and Applications, Volume 2009 (2009), Article ID 676373. 